\title{
Male breast involvement in chronic lymphocytic leukemia. A case report and review of the literature
}

\author{
VASILIKI ANAGNOSTOPOULOU ${ }^{1)}$, Niki MANTHA ${ }^{1)}$, KONSTANTINOS SAPALIDIS ${ }^{2)}$, EIRINI TOLPARIDOU ${ }^{3)}$, \\ ELISAVET GEORGIOU ${ }^{4)}$, TRIANTAFYLLIA KOLETSA ${ }^{1)}$
}

1) Department of Pathology, Faculty of Medicine, Aristotle University of Thessaloniki, Thessaloniki, Greece

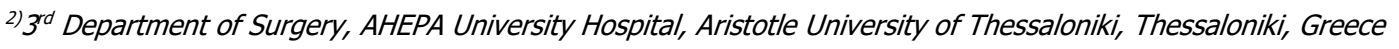

3) Laboratory of Pathology, Mamatseio General Hospital, Kozani, Greece

4) Department of Biological Chemistry, Faculty of Medicine, Aristotle University of Thessaloniki, Thessaloniki, Greece

\begin{abstract}
Male breast lymphoma is a rare extranodal lymphoma occupying the mammary gland, and it could be either primary or secondary. A 78-yearold man presented an enlargement of the right breast. He had no medical history of interest. On physical examination, a unilateral, painless breast lump was found, with no skin changes or nipple discharge. There was no palpable lymphadenopathy. Routine laboratory tests revealed leukocytosis and lymphocytosis. Excisional biopsy of the breast lesion revealed mammary tissue infiltration by chronic lymphocytic leukemia (CLL) with plasmacytoid features and immunoglobulin G/kappa monotypic expression. To our knowledge, this is the first report of male breast involvement by CLL. Considering important the collection of clinicopathological data of all reported male breast lymphoma cases, a literature review is presented.
\end{abstract}

Keywords: breast lymphoma, male breast, CLL, NHL, plasmacytoid differentiation of CLL.

\section{Introduction}

Lymphomas rarely involve the breast gland and the majority of them are non-Hodgkin's lymphomas (NHL), whereas Hodgkin's lymphomas are presented in the literature only as exceptional cases [1]. NHL of the breast is an uncommon disease with primary breast lymphoma (PBL) having a reported incidence from $0.04 \%$ to $0.5 \%$ and with secondary breast lymphoma an incidence of $0.07 \%$ [2]. The majority of breast lymphomas are of B-cell origin [2]. In the literature, most of the cases presented with breast lymphoma correspond to female individuals, whereas male patients were only few. Diagnosis of breast lymphoma by imaging modalities may be difficult as there are no specific features [3] and fine-needle aspiration (FNA) or excisional biopsy is recommended to reach the correct diagnosis.

\section{Aim}

A case of a male patient complaining of a breast lump, which proved to be a secondary involvement of a systematic lymphoma, is herein presented while reviewing the primary and secondary male breast lymphomas, which are reported or referred to in English literature.

\section{$\square$ Case presentation}

A 78-year-old man, with no medical history of interest, presented an enlargement of the right breast. On physical examination, the enlargement was unilateral, painless, with no skin changes or nipple discharge and no palpable lymphadenopathy. The patient was otherwise asymptomatic and did not complain about loss of weight, sweating or fever. Chest X-ray did not show any lesion. Routine laboratory investigation revealed remarkable increase of white blood cells $57640 / \mu \mathrm{L}$, with $77.2 \%$ lymphocytes, $11.9 \%$ neutrophils, $10.1 \%$ monocytes. There was no anemia, thrombocytopenia, or any lymphadenopathy or hepatosplenomegaly on physical examination. Immunophenotyping of peripheral blood lymphocytes by flow cytometry identified a kappa monoclonal B-cell population. B-cells were expressing cluster of differentiation (CD) 20, CD5, CD23 and were negative for CD79b and FMC7. The patient underwent excisional biopsy, which was preferable to him than the FNA option, since he insisted on the lump surgical removal. A partial mastectomy specimen was sent for histological examination. Immunohistochemistry was performed on serial fresh cut $3-\mu$ m thick unstained paraffin sections for CD20 (clone L-26, Dako, Glostrup, Denmark), paired box protein 5 (PAX5) (clone DAKPax-5, DakoCytomation, Glostrup, Denmark), CD3 (clone NCL-CD3-PS1, Novocastra, Newcastle, UK), CD5 (clone SP19, Spring Bioscience, UK), CD23 (clone SP23, Spring Bioscience), CD10 (clone 56C6, Novocastra), B-cell lymphoma (Bcl) 2 (clone 124, DakoCytomation), Bcl-6 (clone PG-B6p, DakoCytomation), cyclin D1 (clone P2D11F11, Novocastra), CD43 (clone DFT-1, Dako Cytomation), CD138 (clone MI15, DakoCytomation), immunoglobulin (Ig) M (polyclonal, DakoCytomation), IgG (polyclonal, DakoCytomation), IgA (polyclonal, Dako Cytomation), $\kappa$ (clone kappa light chains, DakoCytomation), $\lambda$ (clone lambda light chains, DakoCytomation), Ki67 (clone MIB-1, DakoCytomation). The immunostaining was performed on automated immunostainers, namely Bond Max (Leica Microsystems, Wetzlar, Germany) and Dako Autostainer (Dako, Denmark).

This is an open-access article distributed under the terms of a Creative Commons Attribution-NonCommercial-ShareAlike 4.0 International Public License, which permits unrestricted use, adaptation, distribution and reproduction in any medium, non-commercially, provided the new creations are licensed under identical terms as the original work and the original work is properly cited. 
The surgical specimen measured $3 \times 2 \mathrm{~cm}$. On cut sections, a $2.5 \times 2 \mathrm{~cm}$ tan-white colored lesion, with no well-circumscribed borders was observed within the adipose tissue. Histological examination revealed breast tissue infiltration by large nodular foci of lymphoid cells (Figure 1A). The tumor cells were small to medium-sized with roundish nuclei, indistinct nucleoli and scanty mitotic activity. There were also larger cells with morphological features of prolymphoid lymphocytes and paraimmunoblasts, singly or in small aggregates similar to proliferation centers. In addition, large aggregates of cells having plasmacytoid features were found (Figure 1B).

Immunohistochemical analysis revealed positivity of the neoplastic lymphoid cells for CD20, PAX5 (Figure 1C), Bcl-2, CD5 (Figure 1D), CD23 (Figure 1E), CD43, whereas immunostainings for CD3, CD10, cyclin D1, Bcl-6 and CD138 were negative. The proliferation index was approximately $20 \%$. The plasmacytoid cells showed monotypic kappa light (Figure 1F) and IgG heavy chain expression.
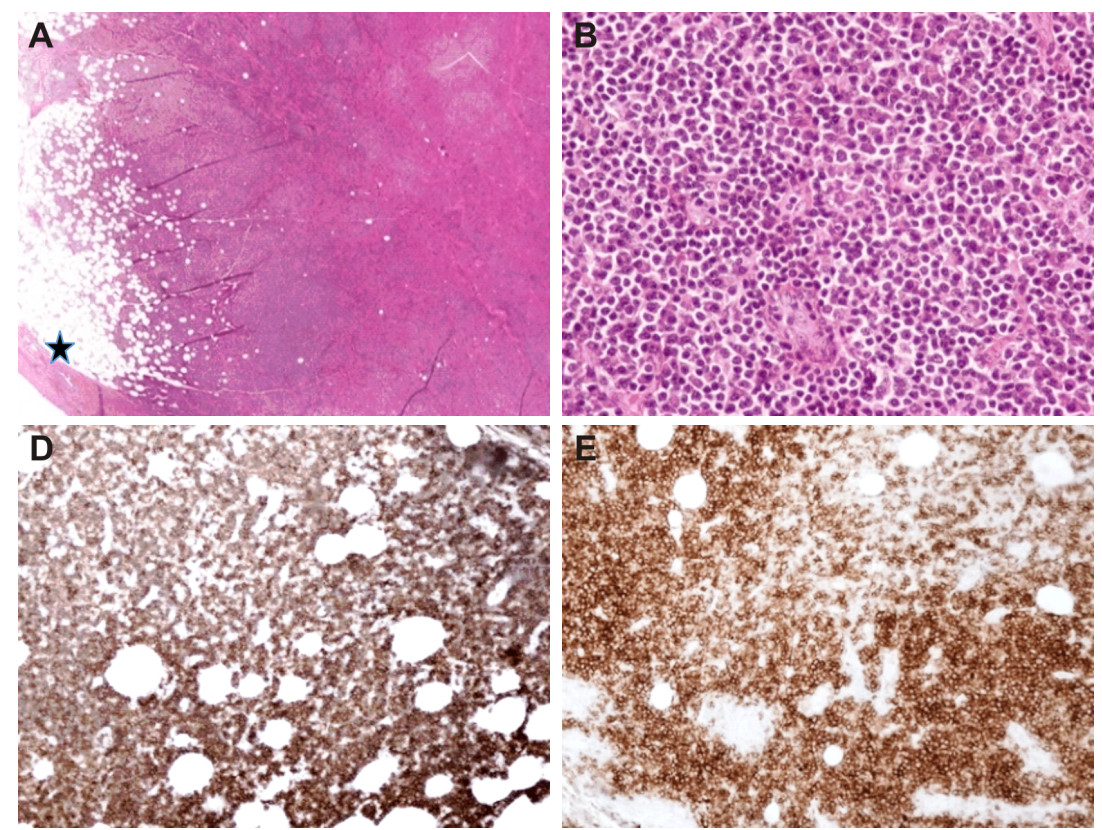
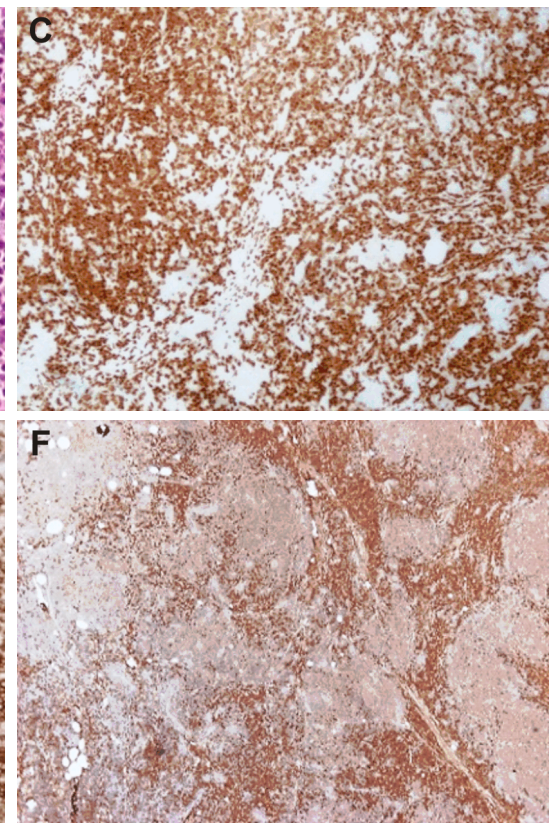

Figure 1 - Breast tissue infiltration by neoplastic lymphoid population (A, asterisk: mammary duct), consisting of small to medium-sized cells (B), some of which present plasmacytoid features (B), immunoreactive to PAX5 (C), CD5 (D), $C D 23(E)$ and kappa light chains $(F)$ antibodies. HE staining: $(A) \times 20 ;(B) \times 400$. Immunohistochemistry: $(C-E) \times 100$; (F) $\times$ 40. PAX5: Paired box protein 5; CD: Cluster of differentiation; HE: Hematoxylin-Eosin.

In summary, histopathological and immunohistochemical findings confirmed the diagnosis of a B-cell chronic lymphocytic leukemia (CLL) with plasmacytoid differentiation and $\mathrm{IgG} / \kappa$ monotypic expression.

The patient underwent computed tomography (CT) scan without any evidence of lymphadenopathy or organomegaly or signs of other organ involvement. According to Rai staging system, the disease was characterized as IAE.

\section{• Discussions}

The peculiarity of this case lies in the fact that a breast lump led to the diagnosis of CLL. The patient had no other symptoms and no other tissue involvement by the lymphoma. Routine laboratory test during breast lump investigation revealed a striking leukocytosis with lymphocytosis. However, in the absence of lymphadenopathy, organomegaly and symptoms, the possibility of breast tissue involvement by lymphoma was unexpected. CLL involvement of the breast is extremely rare in females $[4,5]$ and this is the first reported case in males.

CLL is a common low-grade B-cell NHL that could be presented at several extranodal anatomical sites. Leukocytosis with lymphocytosis is characteristic finding of the disease. CLL exhibits a male predominance and affects mainly middle-aged to elderly patients [6], who are commonly asymptomatic at the time of the diagnosis, as observed in our case, as well. An interesting feature of this case is the presence of plasmacytoid differentiation of the lymphoma. CLL cases consisting of lymphocytes with up to $25 \%$ or more having a plasmacytoid form [7] are considered a distinct morphological variant of this type of lymphoma, sometimes raising differential diagnostic problems with lymphoplasmacytic lymphoma. However, the distinctive CD5+, CD23+, cyclin D1- immunophenotype and the presence of prolymphocytes and paraimmunoblasts support the diagnosis of CLL. Whether the microenvironment is responsible for such a differentiation remains to be clarified.

Breast lymphoma is a rare breast malignancy even in females and can be either primary or secondary [8, 9]. The clinical criteria for the classification of PBL include adequate pathological evaluation with mammary tissue in close association with lymphomatous infiltrate, without evidence of disseminated lymphoma or a previous history of lymphoma [10]. According to the literature, secondary breast lymphoma is more frequent than PBL that varies from $0.85 \%$ to $2.2 \%$ of all extranodal malignant lymphomas [11]. The median age of patients ranges from 60 to 65 years [12]. Bilateral breast involvement occurs in $11 \%$ of all breast lymphomas [13]. Diffuse large B-cell lymphoma is the most common histological subtype [10]. 
The clinical manifestation usually includes a painless breast mass, most often presented in the external superior quadrant. Other symptoms/signs may be palpable lymph nodes, local pain, and inflammation [14]. Skin changes, edema and local pain usually suggest a T-cell lymphoma. As far as the imaging findings are concerned, the lymphoma on mammography appears as a solitary, noncalcified, circumscribed or not, oval or round mass. Ultrasonography is more informative about lesions that present themselves as diffuse infiltration on mammography or lesions associated with skin thickening or edema. The mass on CT appears circumscribed round or oval while the value of magnetic resonance image (MRI) is not established yet [14].

Lymphoma of the male breast is extremely rare and only 37 cases have been reported or referred in the English literature, so far. Clinicopathological data of the reported cases, including the present one, are summarized in Table 1.

Table 1 - Clinicopathological characteristics of male breast lymphoma cases

\begin{tabular}{|c|c|c|c|c|c|c|c|c|}
\hline No. & $\begin{array}{c}\text { Age } \\
\text { [years] }\end{array}$ & $\begin{array}{c}\text { Size of tumor } \\
{[\mathrm{cm}]}\end{array}$ & $\begin{array}{l}\text { Site of } \\
\text { tumor }\end{array}$ & $\begin{array}{c}\text { Clinical } \\
\text { information }\end{array}$ & $\begin{array}{l}\text { Lymph } \\
\text { nodes }\end{array}$ & Histology & $\begin{array}{l}\text { Primary I } \\
\text { secondary }\end{array}$ & $\begin{array}{l}\text { Author(s), year } \\
\text { [Ref. No.] }\end{array}$ \\
\hline 1. & 9 & N/A & $\mathrm{N} / \mathrm{A}$ & N/A & $\mathrm{N} / \mathrm{A}$ & $\mathrm{N} / \mathrm{A}$ & Primary & Docimo, 1959 [14] \\
\hline 2. & 35 & $\mathrm{~N} / \mathrm{A}$ & $\mathrm{R}$ breast & $\mathrm{N} / \mathrm{A}$ & $\mathrm{N} / \mathrm{A}$ & $\mathrm{N} / \mathrm{A}$ & Primary & Sinner, 1961 [14] \\
\hline 3. & 38 & $\mathrm{~N} / \mathrm{A}$ & $\mathrm{R}$ breast & $\mathrm{N} / \mathrm{A}$ & $\mathrm{N} / \mathrm{A}$ & $\mathrm{N} / \mathrm{A}$ & Primary & $\begin{array}{c}\text { Bettini \& Saint Omer } \\
1964[14]\end{array}$ \\
\hline 4. & 81 & $4 \times 4$ & $\mathrm{R}$ breast & N/A & Present & B-cell, DSNC & Primary & Aoi et al., 1985 [14] \\
\hline 5. & 65 & $4 \times 5 \times 2$ & $\mathrm{~L}$ breast & $\mathrm{N} / \mathrm{A}$ & Absent & B-cell, DLBCL & Primary & $\begin{array}{c}\text { Yamamoto et al., } \\
1986[14]\end{array}$ \\
\hline 6. & 74 & $>2$ & $\mathrm{R}$ breast & N/A, prostatectomy & Present & B-cell, DLBCL & Primary & $\begin{array}{c}\text { Kawanisi et al., } \\
1989[14]\end{array}$ \\
\hline 7. & 81 & $\mathrm{~N} / \mathrm{A}$ & $\begin{array}{l}\text { Bilateral, } \\
\text { diffuse }\end{array}$ & $\begin{array}{c}\text { Gynecomastia, } \\
\text { suspicious of prostatic } \\
\text { nodule, Estrogen TX } \\
\text { (9 years) }\end{array}$ & Present & B-cell, DLBCL & Primary & $\begin{array}{c}\text { Hugh et al., } 1990 \\
\text { [15] }\end{array}$ \\
\hline 8. & 65 & $7 \times 5 \times 5$ & $\mathrm{~L}$ breast & N/A & Absent & B-cell, DLBCL & Primary & $\begin{array}{c}\text { Kitaoka et al., } 1900 \\
{[14]}\end{array}$ \\
\hline 9. & 45 & $\begin{array}{l}5.6 \times 5 \times 3.5 / \\
4 \times 3.5 \times 2.2\end{array}$ & $\begin{array}{c}\text { Bilateral, } \\
\text { under nipple }\end{array}$ & N/A & Absent & B-cell, DLBCL & Primary & $\begin{array}{c}\text { Murakami et al., } \\
1993 \text { [14] }\end{array}$ \\
\hline 10. & 69 & $11 \times 11 \times 8$ & $\mathrm{R}$ breast & $\begin{array}{c}\text { N/A, Estrogen TX } \\
\text { (10 years) }\end{array}$ & Absent & B-cell, DM & Primary & $\begin{array}{c}\text { Okada et al., } 1995 \\
{[14]}\end{array}$ \\
\hline 11. & $\mathrm{~N} / \mathrm{A}$ & $\mathrm{N} / \mathrm{A}$ & $\mathrm{N} / \mathrm{A}$ & $\mathrm{N} / \mathrm{A}$ & $\mathrm{N} / \mathrm{A}$ & DLBCL & Primary & $\begin{array}{c}\text { Abbondanzo et al., } \\
1996[16]\end{array}$ \\
\hline 12. & $\mathrm{~N} / \mathrm{A}$ & $\mathrm{N} / \mathrm{A}$ & $\mathrm{N} / \mathrm{A}$ & $\mathrm{N} / \mathrm{A}$ & $\mathrm{N} / \mathrm{A}$ & DLBCL & Primary & $\begin{array}{c}\text { Abbondanzo et al., } \\
1996 \text { [16] }\end{array}$ \\
\hline 13. & 76 & $5.6 \times 5.5 \times 3.5$ & $\mathrm{R}$ breast & Gynecomastia & Absent & B-cell, DLBCL & Primary & $\begin{array}{c}\text { Murata et al., } 1996 \\
{[17]}\end{array}$ \\
\hline 14. & 65 & $4 \times 5 \times 2.5$ & $\mathrm{~L}$ breast & Elastic-hard mass & Present & B-cell, DLBCL & Primary & $\begin{array}{c}\text { Hinoshita et al., } \\
1998 \text { [18] }\end{array}$ \\
\hline 15. & 69 & $2.4 \times 3.9$ & $\mathrm{~L}$ breast & $\begin{array}{c}\text { Palpable mass, } \\
\text { bilateral gynecomastia }\end{array}$ & Absent & B-cell, DLBCL & Primary & $\begin{array}{c}\text { Sashiyama et al., } \\
1999[14]\end{array}$ \\
\hline 16. & $\mathrm{~N} / \mathrm{A}$ & $\mathrm{N} / \mathrm{A}$ & $\mathrm{N} / \mathrm{A}$ & $\mathrm{N} / \mathrm{A}$ & $\mathrm{N} / \mathrm{A}$ & $N / A$ & $\mathrm{~N} / \mathrm{A}$ & $\begin{array}{c}\text { Wong et al., } 2002 \\
{[19]}\end{array}$ \\
\hline 17. & 44 & 2 & $\mathrm{~L}$ breast & Palpable mass & Present & B-cell, DLBCL & Primary & $\begin{array}{c}\text { Cabras et al., } 2004 \\
{[20]}\end{array}$ \\
\hline 18. & 67 & $6 \times 5 \times 4$ & $\mathrm{R}$ breast & Palpable mass & Present & B-cell, DLBCL & Primary & $\begin{array}{c}\text { Mpallas et al., } 2004 \\
{[21]}\end{array}$ \\
\hline 19. & 50 & $4 \times 4 \times 7$ & $\mathrm{R}$ breast & $\begin{array}{c}\text { Palpable breast mass, } \\
\text { axillary masses }\end{array}$ & Present & $\begin{array}{l}\text { B-cell, DLBCL } \\
\text { (ARL) }\end{array}$ & Primary & $\begin{array}{c}\text { Chanan-Khan et al., } \\
2005 \text { [22] }\end{array}$ \\
\hline 20. & 63 & $\mathrm{~N} / \mathrm{A}$ & $\mathrm{R}$ breast & Palpable mass & Present & DLBCL & Secondary & $\begin{array}{c}\text { Vignot et al., } 2005 \\
{[23]}\end{array}$ \\
\hline 21. & 33 & $\mathrm{~N} / \mathrm{A}$ & $\mathrm{L}$ breast & Breast mass & $\mathrm{N} / \mathrm{A}$ & TCRBCL & Secondary & $\begin{array}{c}\text { Duncan et al., } 2006 \\
{[2]}\end{array}$ \\
\hline 22. & 65 & 3 & $\mathrm{R}$ breast & Palpable mass & Absent & T-cell, ALCL & Primary & $\begin{array}{c}\text { Gualco et al., } 2009 \\
{[24]}\end{array}$ \\
\hline 23. & 64 & $6 \times 7$ & $\mathrm{~L}$ breast & Painless growing lump & Absent & B-cell, DLBCL & Primary & $\begin{array}{c}\text { Miura et al., } 2009 \\
{[25]}\end{array}$ \\
\hline 24. & 62 & $7 \times 4$ & $\mathrm{~L}$ breast & Palpable breast mass & Present & B-cell, MZL & Primary & $\begin{array}{c}\text { Duman et al., } 2011 \\
{[26]}\end{array}$ \\
\hline 25. & 51 & 2.3 & L breast & Palpable mass & Absent & T-cell, ALCL & Primary & Ko et al., 2012 [27] \\
\hline 26. & 76 & $\mathrm{~N} / \mathrm{A}$ & L breast & Palpable lumps & Absent & B-cell, DLBCL & Primary & $\begin{array}{c}\text { Mouna et al., } 2012 \\
{[28]}\end{array}$ \\
\hline 27. & 48 & $4.8 \times 4.7$ & $\mathrm{R}$ breast & $\begin{array}{l}\text { Painful lump, HIV- } \\
\text { positive, ART-induced } \\
\text { gynecomastia }\end{array}$ & Present & B-cell, DLBCL & Primary & $\begin{array}{c}\text { Rastogi et al., } 2012 \\
\text { [29] }\end{array}$ \\
\hline 28. & 85 & $\mathrm{~N} / \mathrm{A}$ & $\mathrm{R}$ breast & Palpable mass & $\mathrm{N} / \mathrm{A}$ & B-cell lymphoma & N/A & $\begin{array}{c}\text { Surov et al., } 2012 \\
{[8]}\end{array}$ \\
\hline 29. & 46 & $3 \times 4$ & $\mathrm{R}$ breast & Palpable mass & Present & DLBCL & Primary & $\begin{array}{c}\text { Lokesh et al., } 2013 \\
{[30]}\end{array}$ \\
\hline 30. & 60 & $5 \times 4$ & $\mathrm{~L}$ breast & Palpable mass & Present & SLL & Primary & $\begin{array}{l}\text { Lokesh et al., } 2013 \\
{[30]}\end{array}$ \\
\hline
\end{tabular}




\begin{tabular}{|c|c|c|c|c|c|c|c|c|}
\hline No. & $\begin{array}{c}\text { Age } \\
\text { [years] }\end{array}$ & $\begin{array}{c}\text { Size of tumor } \\
{[\mathrm{cm}]}\end{array}$ & $\begin{array}{l}\text { Site of } \\
\text { tumor }\end{array}$ & $\begin{array}{c}\text { Clinical } \\
\text { information }\end{array}$ & $\begin{array}{l}\text { Lymph } \\
\text { nodes }\end{array}$ & Histology & $\begin{array}{l}\text { Primary I } \\
\text { secondary }\end{array}$ & $\begin{array}{l}\text { Author(s), year } \\
\text { [Ref. No.] }\end{array}$ \\
\hline 31. & 50 & $2 \times 2$ & $\mathrm{~L}$ breast & $\begin{array}{l}\text { Gynecomastia, } \\
\text { palpable lump } \\
\text { behind nipple }\end{array}$ & Present & B-cell, DLBCL & Primary & $\begin{array}{c}\text { Mukhtar et al., } 2013 \\
\text { [31] }\end{array}$ \\
\hline 32. & 70 & $\mathrm{~N} / \mathrm{A}$ & $\mathrm{R}$ breast & $\begin{array}{l}\text { Bilateral enlargement, } \\
\mathrm{R} \text { breast induration }\end{array}$ & Absent & $\begin{array}{l}\text { B-cell recurrence } \\
\text { of } \mathrm{MCL}\end{array}$ & Secondary & $\begin{array}{c}\text { Ikeda et al., } 2015 \\
{[3]}\end{array}$ \\
\hline 33. & 56 & $4 \times 2.5 \times 3.7$ & $\mathrm{R}$ breast & Palpable mass & Present & B-cell, MCL & Secondary & $\begin{array}{c}\text { Ruiz Pardo et al., } \\
2017 \text { [32] }\end{array}$ \\
\hline 34. & $\mathrm{~N} / \mathrm{A}$ & $\mathrm{N} / \mathrm{A}$ & $\mathrm{N} / \mathrm{A}$ & N/A & & B-cell, aggressive & Primary & $\begin{array}{c}\text { Franco Pérez, } 2017 \\
{[33]}\end{array}$ \\
\hline 35. & 65 & $1.7 \times 1.5$ & $\mathrm{R}$ breast & $\begin{array}{c}\text { Cervical } \\
\text { lymphadenopathy, } \\
\text { gynecomastia }\end{array}$ & Present & B-cell, DLBCL & Secondary & Sharma, 2017 [34] \\
\hline 36. & 74 & $\mathrm{~N} / \mathrm{A}$ & $\mathrm{R}$ breast & N/A & $\mathrm{N} / \mathrm{A}$ & B-cell, DLBCL & Primary & $\begin{array}{c}\text { Tokuyama et al., } \\
2017 \text { [35] }\end{array}$ \\
\hline 37. & 47 & $\mathrm{~N} / \mathrm{A}$ & Bilateral & Gynecomastia & Present & $\mathrm{HL}$ & Secondary & $\begin{array}{c}\text { Lin \& Dalal, } 2018 \\
\text { [1] }\end{array}$ \\
\hline 38. & 78 & $2.5 \times 2$ & $\mathrm{R}$ breast & Breast mass & Absent & CLL & Secondary & Present case \\
\hline
\end{tabular}

N/A: Not available; R: Right; L: Left; HIV: Human immunodeficiency virus; ART: Antiretroviral treatment; DSNC: Diffuse small non-cleaved cell; DLBCL: Diffuse large B-cell lymphoma; DM: Diabetes mellitus; ARL: Acquired immunodeficiency syndrome (AIDS)-related lymphoma; TCRBCL: T-cell/histiocyte-rich B-cell lymphoma; ALCL: Anaplastic large-cell lymphoma; MZL: Marginal zone lymphoma; SLL: Small lymphocytic lymphoma; MCL: Mantle cell lymphoma; HL: Hodgkin's lymphoma; CLL: Chronic lymphocytic leukemia.

In this review, we collected all primary and secondary breast lymphomas in males with infiltration of mammary gland, excluding those of cutaneous breast lymphoma. According to the information that was available for each case, we found that there was a wide range of age distribution from nine to 85 years, with a median age of 60 years, similar to that described in female breast lymphoma. The majority of the cases occurred in the right breast $(18 / 33,54.5 \%)$ than the left breast $(12 / 33,36.4 \%)$. In three cases $(3 / 33,9.1 \%)$, lymphoma was present bilaterally. Histologically, the vast majority of the cases correspond to B-cell lymphomas $(31 / 34,91.2 \%)$, mainly of diffuse large B-cell lymphoma (DLBCL) type (23/34, 67.6\%). Although primary lymphomas seem to be more common $(29 / 36,80.6 \%)$ than secondary $(7 / 36,19.4 \%)$, as mirrored by literature review, we should be cautious to reach a safe conclusion, since PBL cases are more often accepted for publication.

\section{a Conclusions}

Given the rarity of male breast lymphoma, as illustrated by this case review, a high level of clinical suspicion is recommended in order to reach early the correct diagnosis. Male breast lymphoma presents a wide variety of morphological characteristics and even in cases of secondary breast lymphoma, the diagnosis can be really challenging, particularly if the first clinical manifestation is a breast lump, without lymph node involvement.

\section{Conflict of interests}

The authors declare that they have no conflict of interests.

\section{References}

[1] Lin DS, Dalal I. Hodgkin's lymphoma of the breast with involvement of the brachial plexus. Appl Radiol, 2018, 47(4): 24-26.

[2] Duncan VE, Reddy VV, Jhala NC, Chhieng DC, Jhala DN. Non-Hodgkin's lymphoma of the breast: a review of 18 primary and secondary cases. Ann Diagn Pathol, 2006, 10(3):144148. https://doi.org/10.1016/j.anndiagpath.2005.09.018 PMID: 16730308
[3] Ikeda T, Bando H, Iguchi A, Tanaka Y, Tohno E, Hara H. Malignant lymphoma of the breast in a male patient: ultrasound imaging features. Breast Cancer, 2015, 22(2):201-205. https:// doi.org/10.1007/s12282-012-0349-y PMID: 22396322

[4] Famà $F$, Barresi V, Giuffrè G, Todaro $P$, Mazzei $S$, Vindigni $A$, Gioffrè-Florio $M$. An unusual presentation of secondary involvement of B-cell chronic lymphocytic leukemia. A case report. Tumori, 2008, 94(4):617-620. https://doi.org/10.1177/ 030089160809400431 PMID: 18822706

[5] Dialani V, Mani K, Johnson NB. Chronic lymphocytic leukemia involving the breast parenchyma, mimicker of invasive breast cancer: differentiation on breast MRI. Case Rep Med, 2013, 2013:603614. https://doi.org/10.1155/2013/603614 PMID: 24151510 PMCID: PMC3789310

[6] Viswanatha DS, Montgomery KD, Foucar K. Mature B-cell neoplasms: chronic lymphocytic leukemia-small lymphocytic lymphoma, B-cell prolymphocytic leukemia, and lymphoplasmacytic lymphoma. In: Jaffe ES, Harris NL, Vardiman JW, Campo E, Arber DA (eds). Hematopathology. $1^{\text {st }}$ edition, Elsevier-Saunders, Philadelphia, 2011, 221-229.

[7] Giardini R, Piccolo C, Rilke F. Primary non-Hodgkin's lymphomas of the female breast. Cancer, 1992, 69(3):725-735. https:// doi.org/10.1002/1097-0142(19920201)69:3<725::aid-cncr28 20690320>3.0.co;2-c PMID: 1730123

[8] Surov A, Holzhausen HJ, Wienke A, Schmidt J, Thomssen C, Arnold D, Ruschke K, Spielmann RP. Primary and secondary breast lymphoma: prevalence, clinical signs and radiological features. Br J Radiol, 2012, 85(1014):e195-e205. https://doi. org/10.1259/bjr/78413721 PMID: 22665932 PMCID: PMC 3474114

[9] Wiseman C, Liao KT. Primary lymphoma of the breast. Cancer, 1972, 29(6):1705-1712. https://doi.org/10.1002/10 97-0142(197206)29:6<1705::aid-cncr2820290640>3.0.co;2-i PMID: 4555557

[10] Joks M, Myśliwiec K, Lewandowski K. Primary breast lymphoma - a review of the literature and report of three cases. Arch Med Sci, 2011, 7(1):27-33. https://doi.org/10.5114/aoms.20 11.20600 PMID: 22291729 PMCID: PMC3258687

[11] Ganjoo K, Advani R, Mariappan MR, McMillan A, Horning S. Non-Hodgkin lymphoma of the breast. Cancer, 2007, 110(1): 25-30. https://doi.org/10.1002/cncr.22753 PMID: 17541937

[12] Jeanneret-Sozzi W, Taghian A, Epelbaum R, Poortmans $P$, Zwahlen D, Amsler B, Villette S, Belkacémi Y, Nguyen T, Scalliet P, Maingon P, Gutiérrez C, Gastelblum P, Krengli M, Raad RA, Ozsahin M, Mirimanoff RO. Primary breast lymphoma: patient profile, outcome and prognostic factors. A multicentre Rare Cancer Network study. BMC Cancer, 2008, 8:86. https://doi.org/10.1186/1471-2407-8-86 PMID: 18380889 PMCID: PMC2330152

[13] Shim E, Song SE, Seo BK, Kim YS, Son GS. Lymphoma affecting the breast: a pictorial review of multimodal imaging findings. J Breast Cancer, 2013, 16(3):254-265. https://doi.org/ 
10.4048/jbc.2013.16.3.254 PMID: 24155754 PMCID: PMC 3800721

[14] Sashiyama H, Abe Y, Miyazawa Y, Nagashima T, Hasegawa M, Okuyama K, Kuwahara T, Takagi T. Primary non-Hodgkin's lymphoma of the male breast: a case report. Breast Cancer, 1999, 6(1):55-58. https://doi.org/10.1007/BF02966907 PMID: 11091691

[15] Hugh JC, Jackson FI, Hanson J, Poppema S. Primary breast lymphoma. An immunohistologic study of 20 new cases. Cancer, 1990, 66(12):2602-2611. https://doi.org/10.1002/10 97-0142(19901215)66:12<2602::aid-cncr2820661224>3.0.co; 2-u PMID: 2249200

[16] Abbondanzo SL, Seidman JD, Lefkowitz M, Tavassoli FA Krishnan J. Primary diffuse large B-cell lymphoma of the breast. A clinicopathologic study of 31 cases. Pathol Res Pract, 1996 , 192(1):37-43. https://doi.org/10.1016/S0344-0338(96)80128-2 PMID: 8685040

[17] Murata T, Kuroda H, Nakahama T, Goshima H, Shiraishi T, Yatani R. Primary non-Hodgkin malignant lymphoma of the male breast. Jpn J Clin Oncol, 1996, 26(4):243-247. https:// doi.org/10.1093/oxfordjournals.jjco.a023222 PMID: 8765183

[18] Hinoshita E, Tashiro H, Takahashi I, Onohara T, Nishizaki T, Matsusaka T, Wakasugi K, Ishikawa T, Kume K, Yamamoto I, Hirota Y. Primary non-Hodgkin's lymphoma of the breast: a report of two cases. Breast Cancer, 1998, 5(3):309-312. https://doi.org/10.1007/BF02966712 PMID: 11091662

[19] Wong WW, Schild SE, Halyard MY, Schomberg PJ. Primary non-Hodgkin lymphoma of the breast: The Mayo Clinic experience. J Surg Oncol, 2002, 80(1):19-25; discussion 26. https://doi.org/10.1002/jso.10084 PMID: 11967901

[20] Cabras MG, Amichetti M, Nagliati M, Orrù P, Mamusa AM, Angelucci E. Primary non-Hodgkin's lymphoma of the breast: a report of 11 cases. Haematologica, 2004, 89(12):15271528. PMID: 15590406

[21] Mpallas G, Simatos G, Tasidou A, Patra E, Galateros G, Lakiotis G, Papanicolaou S, Mpallas E, Anagnostou D. Primary breast lymphoma in a male patient. Breast, 2004, 13(5):436-438. https://doi.org/10.1016/j.breast.2003.11.002 PMID: 15454203

[22] Chanan-Khan A, Holkova B, Goldenberg AS, Pavlick A, Demopoulos R, Takeshita K. Non-Hodgkin's lymphoma presenting as a breast mass in patients with HIV infection: a report of three cases. Leuk Lymphoma, 2005, 46(8):11891193. https://doi.org/10.1080/10428190500084175 PMID: 16085561

[23] Vignot $S$, Ledoussal $V$, Nodiot $P$, Bourguignat $A$, Janvier $M$, Mounier N, Chérel P, Floiras JL, Turpin F. Non-Hodgkin's lymphoma of the breast: a report of 19 cases and a review of the literature. Clin Lymphoma, 2005, 6(1):37-42. https:// doi.org/10.3816/clm.2005.n.025 PMID: 15989705

[24] Gualco G, Chioato L, Harrington WJ Jr, Weiss LM, Bacchi CE. Primary and secondary T-cell lymphomas of the breast: clinicopathologic features of 11 cases. Appl Immunohistochem Mol Morphol, 2009, 17(4):301-306. https://doi.org/10.1097/PAI Ob013e318195286d PMID: 19318917 PMCID: PMC2739299
[25] Miura Y, Nishizawa M, Kaneko H, Watanabe M, Tsudo M. A male with primary breast lymphoma. Am J Hematol, 2009, 84(3): 191-192. https://doi.org/10.1002/ajh.21292 PMID: 18932237

[26] Duman BB, Sahin B, Güvenç B, Ergin M. Lymphoma of the breast in a male patient. Med Oncol, 2011, 28(Suppl 1):S490S493. https://doi.org/10.1007/s12032-010-9675-0 PMID: 20830532

[27] Ko ES, Seol H, Shin JH, Ko EY. Primary anaplastic lymphoma kinase-negative anaplastic large-cell lymphoma of the breast in a male patient. Br J Radiol, 2012, 85(1012):e79-e82. https:// doi.org/10.1259/bjr/23296454 PMID: 22457412 PMCID: PMC 3486659

[28] Mouna B, Saber B, Tijani EH, Hind M, Amina T, Hassan E. Primary malignant non-Hodgkin's lymphoma of the breast: a study of seven cases and literature review. World J Surg Oncol, 2012, 10:151. https://doi.org/10.1186/1477-7819-10151 PMID: 22800119 PMCID: PMC3490884

[29] Rastogi M, Revannasiddaiah S, Seam RK, Gupta MK, Gupta M. Extranodal lymphoma masquerading as carcinoma of the breast in an HIV-positive male patient. BMJ Case Rep, 2012, 2012:bcr2012007472. https://doi.org/10.1136/bcr-2012-007472 PMID: 23266779 PMCID: PMC4544239

[30] Lokesh K, Sathyanarayanan V, Lakshmaiah K, Suresh T, Lokanatha D, Govinda Babu K, Jacob LA, Babu S. Primary breast lymphoma in males - a report of two cases with a review of the literature. Ecancermedicalscience, 2013, 7:347. https://doi.org/10.3332/ecancer.2013.347 PMID: 24723970 PMCID: PMC3965189

[31] Mukhtar R, Mateen A, Rakha A, Khattak R, Maqsood F. Breast lymphoma presenting as gynecomastia in male patient. Breast J, 2013, 19(4):439-440. https://doi.org/10.1111/tbj. 12136 PMID: 23815269

[32] Ruiz Pardo J, López López V, Blanquer Blanquer M, Cabezas Herrera J, Piñero Madrona A. Mantle cell lymphoma involving the breast in a male patient. Cir Esp, 2017, 95(3):170-172. https://doi.org/10.1016/j.ciresp.2016.06.007 PMID: 27453218

[33] Franco Pérez F, Lavernia J, Aguiar-Bujanda D, Miramón J, Gumá J, Álvarez R, Gómez-Codina J, Arroyo FG, Llanos M, Marin M, Alfaro J, Quero C, Delgado M, Nogales E, Menarguez F, Martinez N, Torrente M, Royuela A, Abreu D, Provencio M. Primary breast lymphoma: analysis of 55 cases of the Spanish Lymphoma Oncology Group. Clin Lymphoma Myeloma Leuk, 2017, 17(3):186-191. https://doi.org/10.1016/ j.clml.2016.09.004 PMID: 27847267

[34] Sharma P. Lymphomatous involvement of male breast in a patient with bilateral gynecomastia: demonstration with ${ }^{18} \mathrm{~F}$ fluorodeoxyglucose positron emission tomography-computed tomography. Indian J Nucl Med, 2017, 32(3):249-250. https:// doi.org/10.4103/ijnm.IJNM_35_17 PMID: 28680222 PMCID: PMC5482034

[35] Tokuyama K, Uemoto Y, Kitagawa S, Ohashi H. [Primary breast diffuse large B-cell lymphoma in a male]. Rinsho Ketsueki, 2017, 58(5):455-457. https://doi.org/10.11406/rinketsu.58.455 PMID: 28592759

\section{Corresponding author}

Triantafyllia Koletsa, Assistant Professor of Pathology, MD, PhD, Department of Pathology, Faculty of Medicine, University Campus, Aristotle University of Thessaloniki, 54124 Thessaloniki, Greece; Phone +302310999245, Fax +302310999229, e-mail: tkoletsa@auth.gr 